\title{
Optimal apodizations for on-axis vector vortex coronagraphs
}

\author{
Kevin Fogarty ${ }^{a}$, Laurent Pueyo $^{b}$, Dimitri Mawet ${ }^{c}$ \\ ${ }^{a}$ Johns Hopkins Universty Department of Physics and Astronomy, 3400 N. Charles St, \\ Baltimore, USA; \\ ${ }^{b}$ Space Telescope Science Institute, 3700 San Martin Dr, Baltimore, USA; \\ ${ }^{c}$ European Southern Observatory, Alonso de Cordova 3107 Vitacura, Santiago, Chile
}

\begin{abstract}
Imaging planets requires instruments capable of dealing with extreme contrast ratios and that have a high resolution. Coronagraphs that can reach a contrast ratio in a neighborhood of $10^{9}$ will be capable of observing Jupiter-like planets, while those with a contrast greater than a benchmark number of $10^{10}$ to within a few $\lambda / D$ of an on-axis star will render possible imaging of Earth-like planets. Plans for achieving this feat have been developed for use on telescopes with unobscured, circularly symmetric apertures. However; given that the next generation of large telescopes are on-axis designs with support structures in the telescope aperture and a central obstruction due to the secondary mirror, it has proven necessary to develop coronagraphic techniques that compensate for obstructions. Pueyo \& Norman (2012) present a possible solution to the problem of the support structures using ACAD. In this paper, we present a coronagraphic design that uses a vector vortex and pupil apodization to compensate for the secondary mirror that could possibly be used in conjunction with a wavefront control system and/or ACAD. This coronagraph is capable of achieving a contrast ratio of at least $10^{10}$ in a working angle of $(1.5-30) \lambda / D$ in conjunction with an on-axis telescope. We can construct our pupil using a classical transmissive apodizer or pupil remapping. We find that the mirror shapes required are relatively simple (requiring $\leq 40$ degrees of freedom to describe) and we expect they will be feasible to manufacture, and potentially even to implement with deformable mirrors. By combining existing high-contrast imaging techniques, we demonstrate that a relatively simple design may be used to image exo-Earths.
\end{abstract}

Keywords: Vector Vortex Coronagraph, On-Axis Telescopes, PIAA, Earthlike Exoplanet Imaging

\section{INTRODUCTION}

With the advent of extremely large telescope designs (e.g. the TMT, E-ELT) and the next generation of space telescopes (JWST, AFTA), there are new opportunities to push the boundaries of high contrast imaging. Of particular interest is the possibility of observing Earth-like planets within the habitable zones of other stars, a problem which is being addressed through the use of adaptive optics techniques and new coronagraphic design. ${ }^{1-3}$ Adapting these designs to accommodate the on-axis telescopes with which they could conceivably be paired is an area of current research. ${ }^{2,4,5}$

Using the Earth and Sun as a point of reference, it is common to demand a contrast ratio of $10^{10}$ at an inner working angle of $\sim \lambda / D$ as goals which a coronagrah must be able to achieve in order to image an Earth-like exoplanet. ${ }^{6-8}$ The contrast ratio is the ratio of the flux from the on-axis source (a star) to the flux from a nearby source (a planet), and determines how much light from the PSF of the on-axis source needs to be rejected to view the nearby source (e.g. the star's PSF needs to be $10^{-10}$ of the star's flux at the position of the planet in order for the planet to be visible). Meanwhile, the inner and outer working angles define the annular region, or 'dark hole', around the on-axis source where a contrast ratio of $10^{10}$ needs to be achieved, so the IWA is effectively the angular resolution of the coronagraph. If an observer were 10 parsec away and observing the Sun, the Sun and Earth would have an angular separation of $0 . " 1$ (or $2 \lambda / D$ for a 2 -meter telescope in the V-band), hence an instrument capable of meeting these criteria would barely be able to observe Earth. ${ }^{8}$

Further author information: (Send correspondence to K. Fogarty)

K. Fogarty: E-mail: kfogarty@pha.jhu.edu

L. Pueyo: E-mail: pueyo@stsci.edu

Space Telescopes and Instrumentation 2014: Optical, Infrared, and Millimeter Wave, edited by Jacobus M. Oschmann, Jr., Mark Clampin, Giovanni G. Fazio, Howard A. MacEwen, Proc. of SPIE Vol. 9143, 914326 • ( 2014 SPIE CCC code: $0277-786 \mathrm{X} / 14 / \$ 18 \cdot$ doi: $10.1117 / 12.2056760$

Proc. of SPIE Vol. $9143914326-1$ 
Coronagraphs for off-axis telescopes, which feature circularly symmetrical apertures without obscuration from a secondary mirror or support structures, have been designed which can achieve these goals (e.g. Trauger et al. $2010 ;{ }^{9}$ for an example prototype see Trauger \& Traub $2007^{10}$ and Trauger et al. 2011 ${ }^{11}$ ). In particular, instruments using a vector vortex mask can theoretically provide perfect on-axis light rejection for an unobstructed aperture. ${ }^{12}$ However; considering the upcoming and proposed ELTs and space telescopes that will not feature off-axis secondary mirrors, it will be necessary to examine designs that account for a central obscuration, as well as structural elements affecting the shape of the aperture. Recently, Pueyo \& Norman $(2012)^{2}$ have explored a use of wavefront control systems called Active Compensation of Aperture Discontinuities (ACAD) which is designed to achieve high contrast imaging on telescopes that have support structures and other discontinuities in the telescope aperture. This system augments the use of coronagraphs that can deal with the obscuration from the secondary mirror.

Coronagraphs that expand on the vector vortex mask concept have been designed to address the central obscuration in the aperture due to the secondary mirror, ${ }^{13,14}$ as have designs that involve changing the apodization at the pupil plane, either through the use of mirrors or apodizing filters. ${ }^{15-17}$ Unfortunately, the parameter space of performance vs. manufacturability is big and a lot reamins to be explored.

In this paper, we discuss an innovation on these techniques that can be used with the ACAD system. A design is presented for a high contrast coronagraph that makes use of a vector vortex phase ramp and apodization at the pupil plane. Our coronagraph is similar in concept to the ring-apodized vortex coronagraph of Mawet et al. 2013. ${ }^{14}$ Instead of analytically deriving a pupil apodization that will cancel out the effect of the secondary mirror like they do, we treat the throughput through the pupil like a weighting function to produce a smooth apodization that suppresses the contribution of the secondary mirror to the PSF. The apodization function at the pupil plane can be achieved through the use of a light blocking classical apodizer; however, the design we show here is simple enough to be produced using mirror shapes. This design can be optimized to produce a working region in the PSF that has a contrast approaching or exceeding $10^{10}$, and can minimize the IWA to within $\frac{1.5 \lambda}{D}$.

This paper is divided into four sections. In section 2, we provide motivation for our design and background on the coronagraphy techniques we combine in the present discussion. Section 3 discusses the coronagraph design and derives the necessary calculations to describe the coronagraph discussed in this paper, and in Section 4, we describe the performance of an optimized coronagraph design that achieves the necessary contrast and IWA. In Section 5 we explore using aspheric pupil remapping mirrors to produce our optimal design.

\section{BACKGROUND}

The main challenge we seek to address in this paper is the design of a coronagraph that is capable of exoplanet imaging in the presence of a circularly symmetrical on-axis secondary mirror. In general, in addition to the secondary mirror, on-axis telescope apertures will be affected by segment gaps in the primary mirror, and support structures holding up the secondary. These patterns in the telescope pupil (called "spiders") present challenges to high-contrast imaging, since they alter the electric field amplitude in the pupil plane on the order of a few percent. ${ }^{2}$ Spiders can be compensated for by a strategy such as $\mathrm{ACAD}^{2}$ to compensate for these small-scale artifacts. By dealing with the large-scale central obscuration, our coronagraph completes the task of correcting for artifacts in the telescope aperture. In what follows, we summarize the concepts we are incorporating in our coronagraph design along with the challenges involved in using them on an obscured aperture.

\subsection{Vortex Coronagraphy}

Vortex coronographs use a phase mask called a vector vortex, which adds an azimuthally dependent phase angle to a field passing through it. ${ }^{12,18,19}$ Summarizing Mawet et al. (2011), a vortex ramp of charge $c$ multiplies the focal plane field due to light through an aperture by $e^{i c \theta}$, where $\theta$ is the azimuthal angle around the aperture. The Fourier transform of this, $F\left[e^{i c \theta} \times \frac{2 J_{1}(r A)}{r A}\right]$ (the field in the Lyot plane), becomes a ring of intensity at $r>A$ where $A$ is the radius of the aperture and falling of as $\left(\frac{A}{r}\right)^{2}$ for charge 2 (for charge 4 this expression becomes $\left.2\left(\frac{A}{r}\right)^{2}-3\left(\frac{A}{r}\right)^{4}\right)$. For higher charges the behavior of the electric field becomes more complex, although 

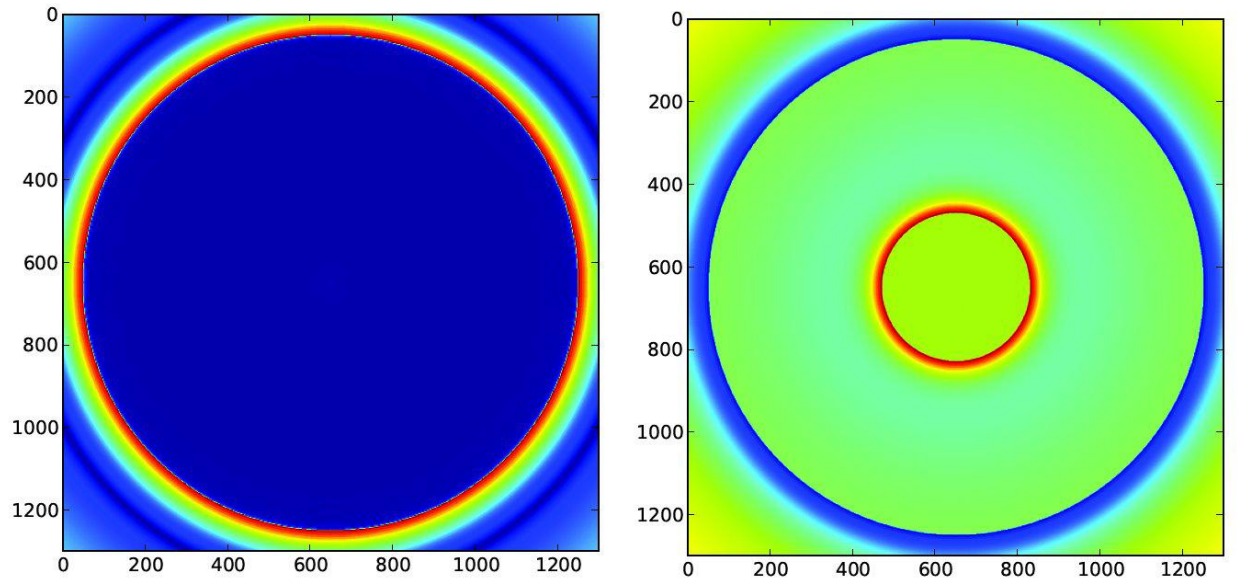

Figure 1. Left Panel Lyot plane of an unobscured vector vortex coronagraph. Right Panel Lyot plane of a centrally obscured vortex coronagraph.

the salient feature that light is pushed into an annulus outside the aperture radius remains true). The light near $A$ is then blocked by a Lyot stop. ${ }^{13}$

For a symmetrical, unobscured aperture, the vortex removes all the light within the aperture, moving it to outside the image of the pupil. This allows coronagraphs to be designed without the need for an obscuring mask in the focal plane, and vortex instruments with high contrast ratios have been designed with IWAs of $\sim 1 \lambda / D .{ }^{20,21}$ For the purposes of imaging exoplanets, this characteristic is appealing, since it allows planets close in to their host stars to be imaged.

The vector vortex is also advantageous with regards to its chromaticity and its sensitivity to pointing errors and measurable stellar sizes. Recently, it has been shown that achromatic vector vortex masks are achievable, ${ }^{22}$ and a broadband vortex with a contrast near $10^{10}$ has been demonstrated in a test bed. ${ }^{23}$ Furthermore, it can be shown that the leakage by the mask of off-axis light goes as $s^{c}$, where $c$ is the charge of the mask and $s$ is the angular position of the light in units of $\lambda / D .^{24,25}$ Therefore, slightly off-axis light due to a small pointing error or finite stellar size contributes limited leakage into the final image.

In the presence of a central obscuration, the vortex ramp is no longer able to exclude light within the central aperture perfectly. On the contrary, a Fourier transform of the focal field $\times e^{i c \theta}$ results in two concentric rings that fall off as $r^{-2}$ (for charge 2); one inside the pupil (see figure 1). This has led to designs such as that in Mawet et al. 2011, ${ }^{13}$ which makes use of two vortex stages to concentrate all of the point source light in a central region where it can be blocked out by an inner Lyot stop. This inner Lyot stop is in the Lyot plane, and so does not affect the IWA of the coronagraph. More recently, Mawet et al. $2013^{14}$ have devised an analytical solution called the ring-apodized vortex coronagraph. This solution involves making an apodizing mask with hard-edged annuli with different throughputs. The intensity rings produced by these discontinuities are matched to the ring produced by the discontinuity of the central obscuration and so cancel them out.

Similarly, we make use of an inner Lyot stop to block out the peak of the light from the inner ring, but instead of using a ring-apodized mask to cancel out the contamination from the secondary mirror, we use a smoothly varying apodization function that can be calculated using numerical optimization. While our innovation does not result in full cancellation of the contaminating light, this light is suppressed sufficiently to image a terrestrial exoplanet. It also provides several advantages both in terms of performance and practicality (see sections 3.2 and 4.1). 


\subsection{Pupil Apodization}

An apodized pupil is constructed by either bending light passing through the pupil plane or by partially blocking it out. We will call methods that involve blocking out or asborbing source flux classical apodization . Classical apodizers exist which have relatively achromatic properties and are suitable for use on a broad-band instrument. ${ }^{4,17,26}$ We will briefly discuss using a classical apodizer for our apodized pupil vortex coronagraph; however, we will focus much of our attention on achieving the same result using shaped mirrors to deflect incident light. The fundamtental design of our coronagraph remains the same regardless of the technique used- but the use of mirrors has a considerable advantage in throughput, so long as they are feasible to make.

Apodizing the pupil can also be achieved through the use of two shaped mirrors. ${ }^{3,27}$ In particular, a system called Phase-Induced Amplitude Apodization (PIAA) has been researched that can provide high contrast and a good IWA when combined with an adaptive optics system (e.g. Guyon $2003,{ }^{3}$ Guyon $2005^{7}$ ). The two mirrors remap the rays of light through an aperture from an on-axis source to produce a specific distribution of light in the pupil, and focus this new beam shape. This approach, which can be used to optimize inner working angle and contrast, does not diminish coronagraph throughput or introduce chromatic effects since it uses mirrors in a regime where ray optics holds. A PIAA system in conjunction with extreme adaptive optics has been implemented on the Subaru Telescope (SCExAO), that has recently begun taking images. ${ }^{28}$

Briefly, the equations that describe how the mirror shapes depend on the desired apodization are:

$$
\begin{gathered}
\frac{\partial \tilde{H}}{\partial \tilde{r}}=\frac{r-\tilde{r}}{P_{0}} \\
\frac{\partial H}{\partial r}=\frac{\partial \tilde{H}}{\partial \tilde{r}} \frac{\partial \tilde{r}}{\partial r},
\end{gathered}
$$

where $\tilde{H}$ and $\tilde{r}$ are the mirror shape and coordinates of the second of the two mirrors, and $H$ and $r$ are the shape and coordinates of the first. $P_{0}$ is a constant related to the distance between the two mirrors. These equations have been simplified for this discussion, and describe circularly symmetric mirrors in the absence of offsets between the two. The dependence of the mirror shapes on the apodization $A$ shows up in $r(\tilde{r})$, which is the inverse of the function mapping rays incident on the first mirror at $r$ to the position $\tilde{r}$ on the second mirror. This function is

$$
r(\tilde{r})=\sqrt{\int_{0}^{\tilde{r}} 2 A^{2}(s) s d s},
$$

which is true for any two-mirror configuration. A detailed derivation of these equations is given in Vanderbei \& Traub 2005. ${ }^{15}$

The main issues with PIAA coronagraphy are the manufacturability of the mirrors and diffraction effects where the curvature becomes severe. These are both the result of the constraints placed upon mirror design when optimizing for the necessary IWA and contrast ratio to image an Earthlike exoplanet. Pluzhnik et al. $2006^{29}$ addresses the problems with PIAA by using classical apodizing masks to reduce the need for highly curved mirror edges and compensate for diffraction.

\section{CORONAGRAPH DESIGN}

\subsection{Principle}

The coronagraph we focus on in this paper has three stages; (A) a pupil plane with an apodizing mask, (B) a focal plane with a vortex phase mask, and (C) a final pupil plane with a Lyot stop. This geometry is summarized in Figure 2. Light from a uniform on-axis point source entering through the telescope aperture into the coronagraph at (A) will have an initial electric field

$$
E_{\text {pup }}=\left[1-\Pi\left(\frac{2 r}{\mu}\right)\right] M(r)
$$




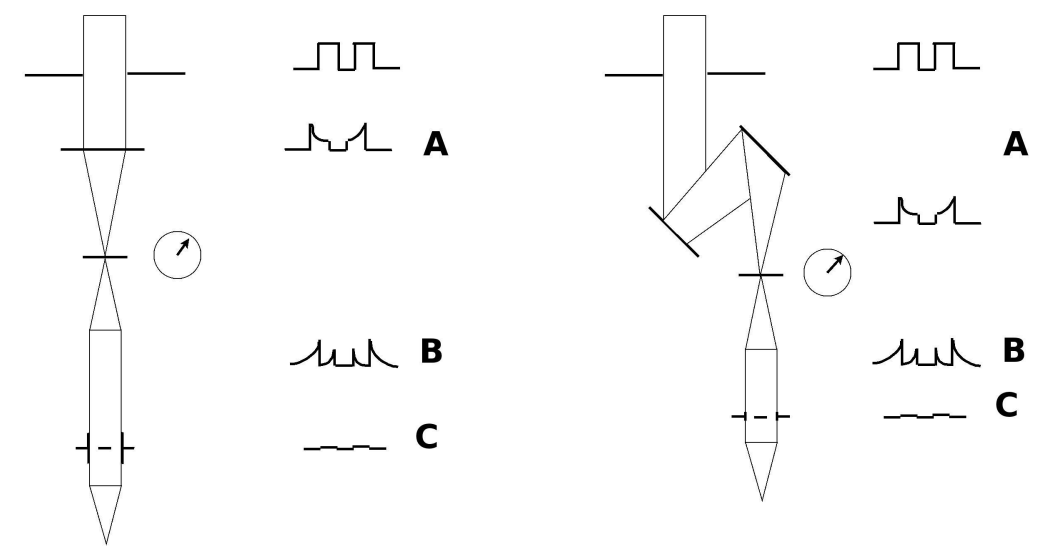

Figure 2. Left: The design of the vector vortex coronagraph is shown schematically, using a classical apodizer to block on-axis light. Right: The same design, except now the apodization of the pupil plane is achieved using a pair of deformable or shaped mirrors.

(A) The light from an on-axis point source in the pupil plane is remapped by the deformable mirrors, producing the desired apodization as a function of radius. The initial aperture, with the central obscuration, is shown at the topmost stage (where the light enters aperture), followed by the shaped aperture after remapping occurs.

(B) A vector vortex mask with a charge c is placed in the focal plane, causing the field to be remapped into two rings. Due to the apodization, the inner ring no longer has an amplitude that falls of as $r^{-2}$.

(C) Inner and outer Lyot stops in the second pupil plane block the sharp peaks in the rings caused by the vector vortex.

where $\mu$ is the percent of the radius obscured by the telescope central obscuration and $M(r)$ the transmission function of the mask. Passing the light through a vortex phase ramp in (B) can be described by two successive Hankel transformations,

$$
E_{V V}(r)=H_{c}^{-1}\left(H_{0}\left(E_{\text {pup }}(r)\right)\right) .
$$

Here, $c$ is the charge of the vortex phase ramp and

$$
\begin{aligned}
H_{n}(f(r)) & =\int_{0}^{\infty} f(r) J_{n}(k r) r d r \\
& =\int_{0}^{\infty} d r f(r) r \frac{1}{2 \pi} \int_{-\pi}^{\pi} e^{-i(n \tau-k r \sin \tau)} d \tau
\end{aligned}
$$

Since $E_{\text {pup }}$ is a circularly symmetric function, $H_{0}\left(E_{\text {pup }}(r)\right)$ is an equivalent operation to the Fourier transform, since

$$
\begin{aligned}
H_{0}(f(r)) & =\frac{1}{2 \pi} \int_{0}^{\infty} \int_{-\pi}^{\pi} d r d \tau f(r) r e^{i k r \sin \tau} \\
& =\frac{1}{2 \pi} \int_{0}^{\infty} \int_{\pi / 2}^{3 \pi / 2} r d r d \theta f(r) e^{i k r \cos \theta} \\
& =\frac{1}{2 \pi} \iint r d r d \theta f(r) e^{i k \cdot r}=F[f(r)]
\end{aligned}
$$

Furthermore, the phase ramp of charge $\mathrm{c}, e^{i c \phi}$, is built right into the definition of the Bessel function $J_{c}(\phi)$. Therefore, when performing the inverse Hankel transformation, the vector vortex is already accomplished in the integrand.

The Lyot stop at (C) consists of both an inner and an outer Lyot stop. The outer Lyot stop performs the same function as it does in the traditional coronagraph. ${ }^{30,31}$ Light from the PSF shifted to the far edge of the pupil plane is blocked out, thereby removing it from the final image (see figure 1). However, the centrally 

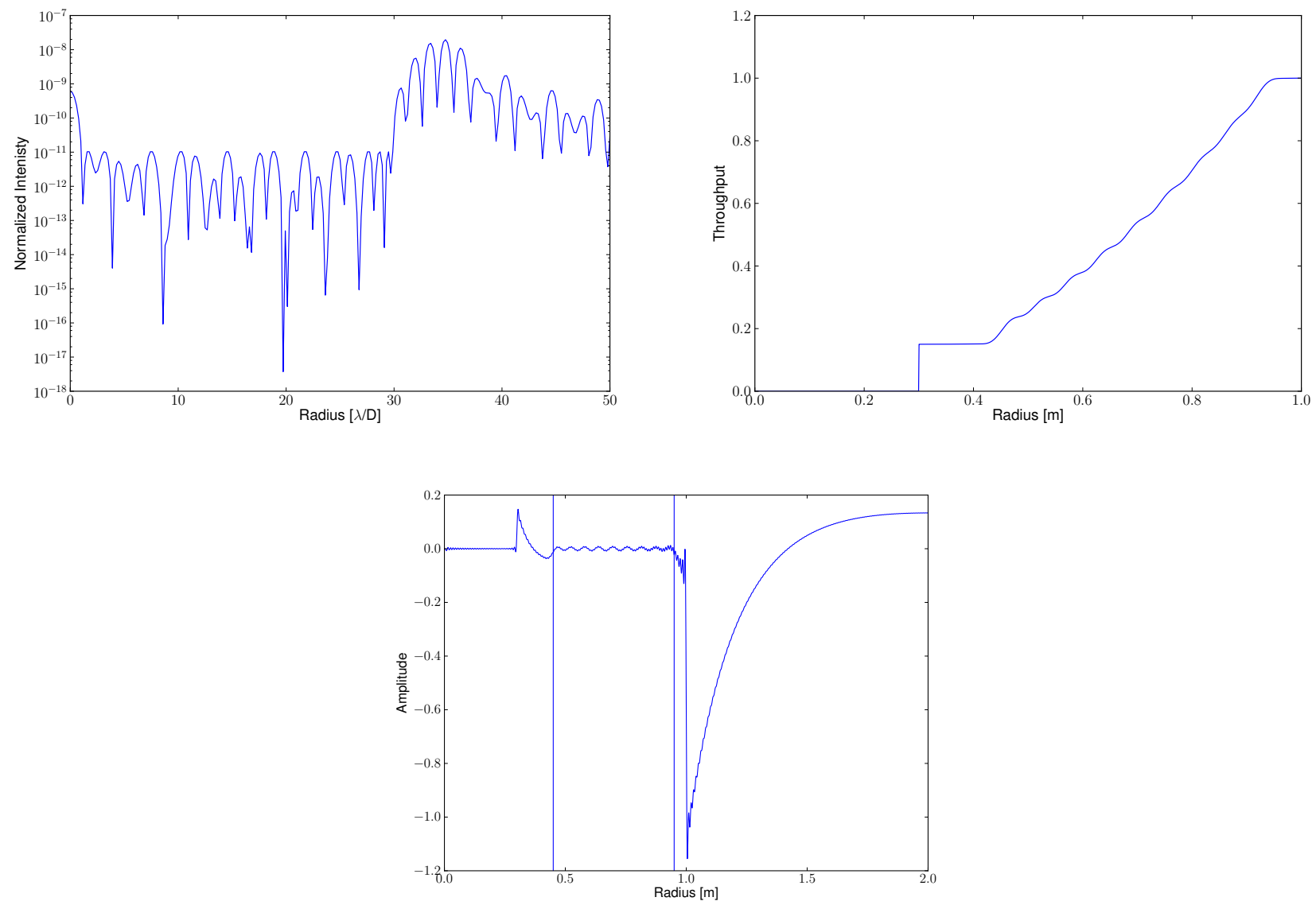

Figure 3. Left: The optimized PSF of the vector vortex coronagraph. Inside the working region, a contrast of $10^{10}$ has been achieved, while outside the IWA and OWA the contrast is lower. Angular distance is expressed in units of $\lambda / D$. Right: The transmission function of the aperture which produces the PSF in the figure above. Since the point source is presume to be on-axis, the aperture transmission function is the product of the central obscuration and the apodizing mask. The mask itself has a transmission which increases monotonically with radius outside the aperture, inside the radius of the central aperture the transmission is unimportant. Bottom: The amplitude of the electric field at the Lyot plane, showing the response of the apdozing mask and vortex. The vertical lines indicate the positions of the inner and outer Lyot stops.

obscured aperture and vortex phase ramp cause there to be a fringe of light near to the center of the Lyot plane (see figure 1). The sharp peak of the fringe is blocked by the inner Lyot stop, while the remaining light from the fringe is minimized by the apodization at the pupil plane.

\subsection{Apodizer Optimization}

Given that the central obscuration in the aperture is fixed, the parameters we can vary include the shape of the pupil mask, the size of the Lyot stops and the charge of the vector vortex. In what follows we hold the Lyot stop sizes and vector vortex charge fixed at values we find produce reasonable results.

We form a basis of mask shapes using functions of the form

$$
F_{n}(r)=\frac{\sqrt{2} J_{10}\left(\frac{Z_{n} r}{R}\right)}{R J_{11}\left(Z_{n}\right)},
$$

where $Z_{n}$ is the nth zero of the Bessel function of order 10. Bessel functions were chosen since the Hankel transform of the pupil mask gives the function's spectrum in terms of Bessel functions, so starting with the 
functions expressed in this basis makes the manipulations described above particularly simple. Bessel functions of order 10 were chosen as the basis in particular, since they are well suited to describing obscured apertures that have zero throughput below some radius. The higher order the Bessel function, the greater the radius is before it has a non-vanishing value, so a basis composed of high-order bessel functions descriping the pupil plane has the central obscuration already 'built in'. The results described below were calculated using the first 32 of these basis functions.

The derivatives and output electric fields for a point source are calculated for each basis function individually. The combination of functions resulting in the mask shape $M(r)$ that optimizes the coronagraph PSF is then solved for, subject to the constraints:

$$
\begin{aligned}
\max [M(r)] & \leq 1 \\
\min [M(r)] & \geq b \\
D[M(r)] & \geq 0,
\end{aligned}
$$

where $b$ sets the minimum throughput.

The constraint on the derivative of the apodizing mask shape $D[M(r)]$, that it be positive, is based on the intuitive notion that the throughput through the mask acts like a weight which can minimize the effect of the central obscuration on the behavior of the vector vortex mask. By demanding that the energy incident upon the vector vortex mask from the outer radii is greater than the energy from the inner radii, this effectively down-weights the central obscuration in the pupil, and makes the pupil look more like an unobscured aperture, with which the vector vortex behaves perfectly. This also allows us to simply constrain the curvature of mirrors producing the apodization, should we implement this apodization with remapping mirrors. Since curvature is strongest where the light rays need to be dispersed the most (i.e. where the throughput is least), the curvature of the mirrors depend on the parameter $b$.

A solution for an optimized pupil mask with a monotonically increasing transmission is shown in figure 3 . The result shown is for an instrument with an inner Lyot stop that covers the inner $0.45 R$ of the pupil and the outer stop covers the outer $0.95 R$, and a charge $c=4$ vector vortex. A central obscuration that covers $30 \%$ of the aperture was assumed. For this setup, we found a result capable of achieving a contrast ratio of at least $10^{10}$ in a dark hole of $(1.5-30) \lambda / D$ could be obtained for $b=0.15$. The resulting PSF is shown in figure 3 .

We use equations 1-3 to plot the mirror shapes in figure 4 which would reproduce this apodization function. We examine these mirrors in more detail in Section 5. Here, we comment that the mirrors exhibit gradual curvature in the region exposed to the on-axis point source, and approach flatness near their outer edges. In Carlotti et al. 2011, ${ }^{32}$ it was shown that the maximum curvature needed in a mirror producing a shaped pupil is proportional to the minimum throughput in the pupil. Since we demand a minimum throughput of $b$ when we find our apodization function, we are equivalently setting a maximum on the curvature of the mirror. Furthermore, the throughput increases with radius and reaches 100\% near the edge of the pupil. Equivalently, the deformation of the shaped mirrors become shallower with increasing radius and becomes flat at the mirrors' outermost radii. This is in contrast to Guyon et al. $2003,{ }^{3}$ which requires mirrors to curve steeply at outer radii and are thus complicated to manufacture. Finally, light from an on-axis source is blocked within the radius of the secondary obscuration, so we do not need to worry about constraining the mirror shape in this region of the shaped mirror.

\section{PERFORMANCE}

\subsection{Tip/Tilt Errors}

Mawet et al. $2010^{24}$ describes the sensitivity of the vector vortex phase mask to tip/tilt error. As stated above, spillover light introduced by telescope tip/tilt is determined by the sensitivity of the vortex to off-axis light, which is approximately $s^{c}$, where $s$ is the distance from the center of the focal plane in $\lambda / \mathrm{D}$ and $\mathrm{c}$ is the charge. In principle, a vector vortex of arbitrarily large charge may be used to immunize the vortex to arbitrarily large tip/tilt errors. However, since we are primarily concerned with devising a coronagraph that incorporates optical elements that are either currently in use or have been examined in a test bed, we limit our attention to a vortex with $c=4 .^{23}$ 


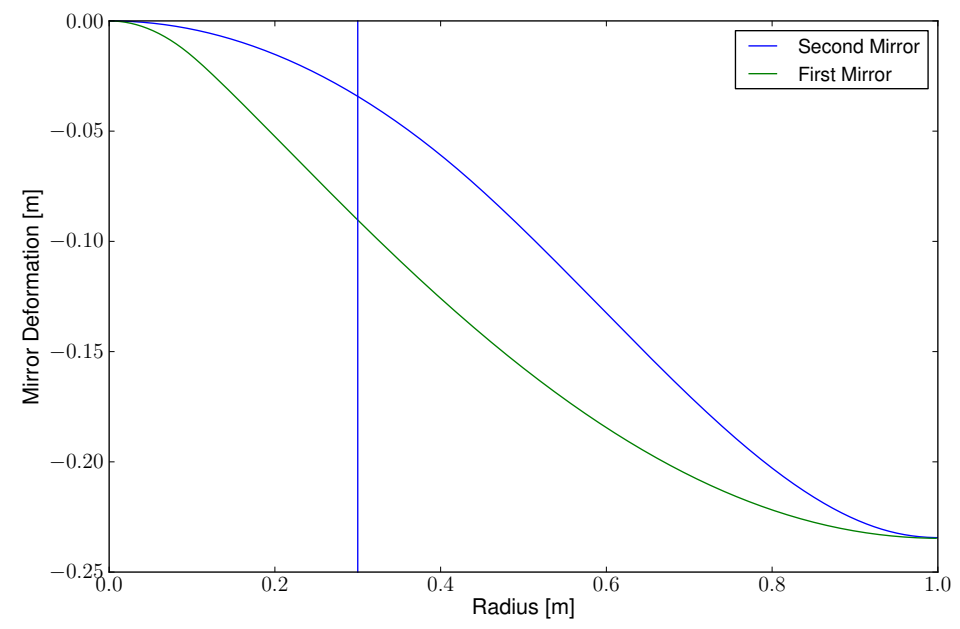

Figure 4. Shapes of the two deformable mirrors required to produce the apodization function in figure 3 . The absolute scale of the deformation is set by the mirror radius $R$ and the distance between the two mirrors $Z$, and the shape of the mirrors scales with the quantity $R^{2} / Z{ }^{3,33}$ Points inward of the blue line on the first mirror see no illumination from an on-axis point source due to the central obscuration. In principle, the deformation of the first mirror could have less curvature than is shown for this region, since it will not reflect any light from a target star.

We examine the impact of introducing a small spot in the center of the vector vortex mask. Not only does this cover defects in the vortex mask that may result from the manufacturing process, the spot should improve the coronagraph's robustness to tip/tilt. This obscuration is equivalently in the center of the image plane, and so effectively reduces the sensitivity of the final image to the position and size of the on-axis point source. We find that we can still recover a PSF with a $>10^{10}$ contrast ratio in our desired dark hole for large spots if we manipulate the value of $b$. If $b=0.1$, then a vector vortex spot with radius $1.0 \lambda / \mathrm{D}$ can be applied, and an optimal apodization function can be found that preserves the inner working angle and contrast.

However, decreasing $b$ comes at the expense of throughput for the off-axis source (ie the exoplanet) if a classical apodizer is used to realize our apodization function, or increased curvature if a shaped mirror is used. With this in mind, we fix $b$ at 0.15 , and use spot with a radius of $0.75 \lambda / \mathrm{D}$. The optimal apodization for these parameter choices preserves a PSF with an IWA of $1.5 \lambda / \mathrm{D}$ and a contrast ratio in the dark hole that is within a factor of 2 of $10^{10}$ (shown in Figure 5). Therefore, we can block out the image of a central source out to a radius of half of our intended IWA, and preserve our dark hole. While further investigation is needed to quantify the effects of adding a spot on tip/tilt error and on using the vector vortex on spatially resolved stars, we expect it will diminish contrast degradation of the PSF due to their extended angular size and positional uncertainty significantly.

\subsection{Throughput}

Figure 6 displays the throughput of an off-axis source as a function of radial distance, both for apodization achieved using a classical apodizer and apodization achieved using shaped mirrors (discussed in Section 5.2 below). If we define the effective IWA of the coronagraph as the position where the off-axis source has half the throughput of maximum, then the IWA for a classical apodizer is $\sim 2.5 \lambda / D$, or $\sim 1.5 \lambda / D$ if mirrors are used. This is close to the design limit of $1.5 \lambda / D$ we set for the IWA when optimizing the apodization function. Using a classical apodizer, we achieve an off-axis throughput of $31 \%$, which compares favorably with the $22 \%$ throughput of the charge 4 ring apodized vortex coronagraph for a central obscuration of $0.3 R_{\text {mirror }}$. While the apodization function we calculated improves on off-axis throughput compared to the charge 4 ring apodized vortex coronagraph when an apodizing mask is used, the throughput is obviously substantially higher if we employ shaped or deformable mirrors to replace the classical apodizer. In that case, a throughput of nearly 

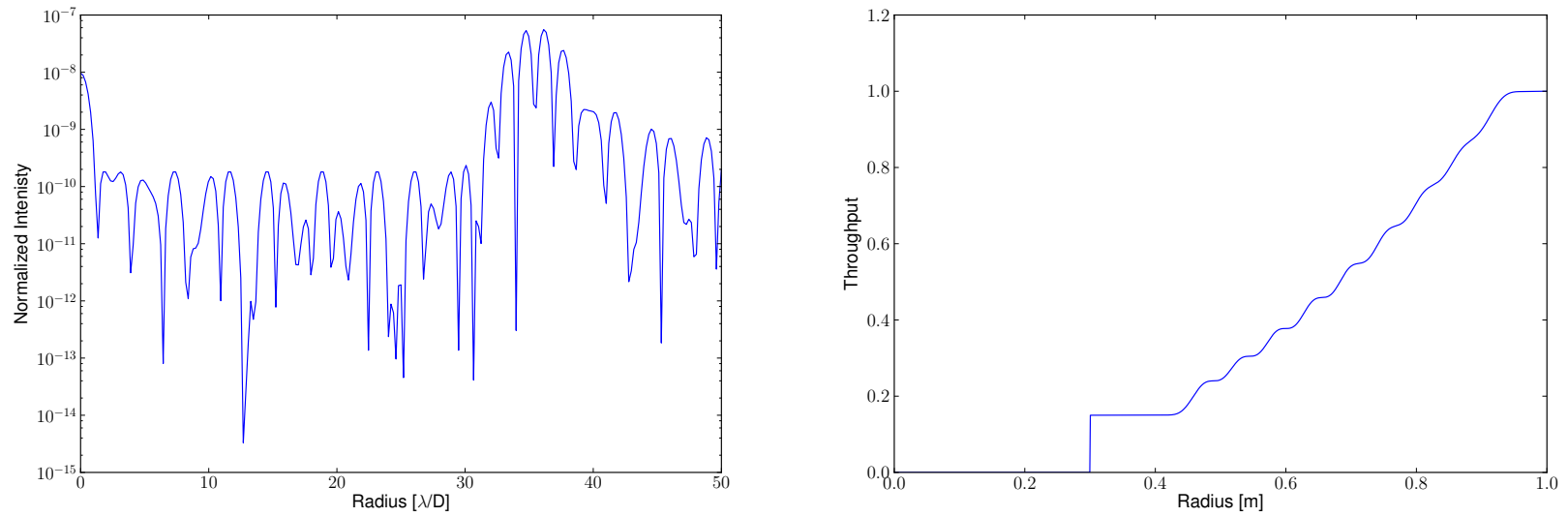

Figure 5. Left: PSF of the coronagraph designed, modified to include an obscuring spot on the vector vortex mask. The spot has a radius of $0.75 \lambda / \mathrm{D}$ and is circularly symmetrical. The dark hole achieved using this design falls short of a contrast ratio exceeding $10^{10}$; however, it remains within a factor of 2 of this benchmark. Right: The optimal apodization function is qualitatively similar to the function shown in Figure 3.

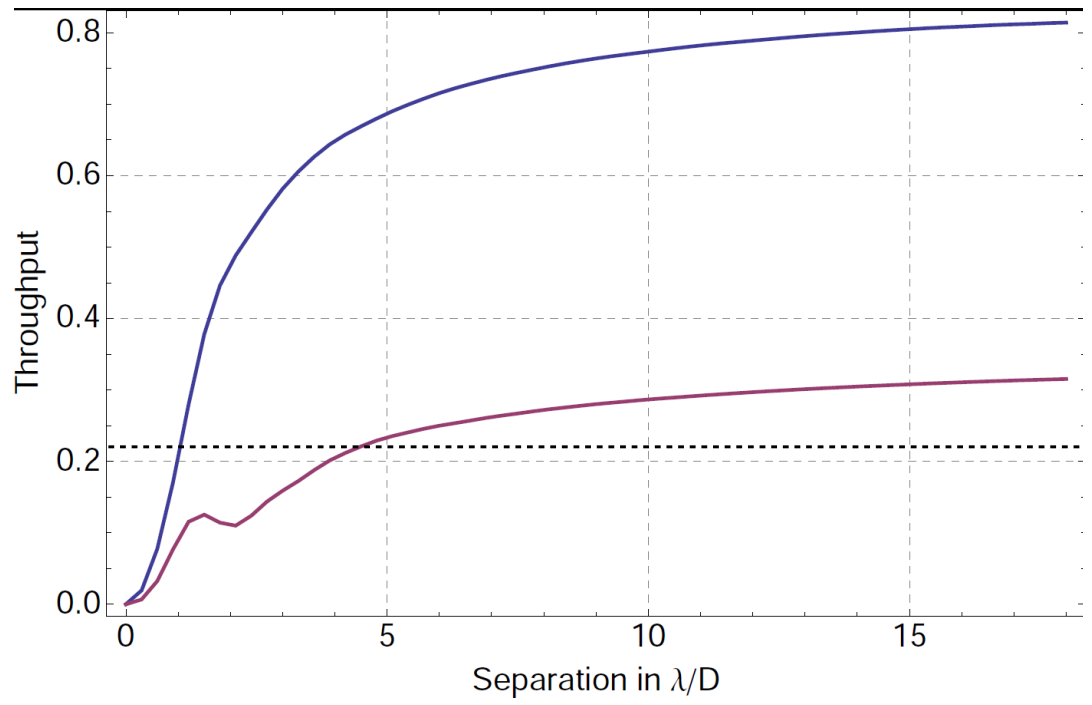

Figure 6. The throughputs for flux from an off-axis point source is shown as a function of separation from the on-axis point source for two versions of our coronagraph design. The maximum off-axis throughput of the system in the dark hole comes out to about 0.31 . The horizontal dashed line represents the maximum throughput of the charge 4 ring-apodized vortex coronagraph $(\sim 0.22)$ assuming a central obscuration of 0.3 . The bottom (purple) curve shows the throughput that is obtained if apodization is achieved using a classical apodizer, which partially or entirely absorbs incident flux. The top (blue) curve shows throughput that is obtained if shaped mirrors are used instead. In this design, loss of throughput comes primarily from the Lyot stops.

$80 \%$ can be achieved for mirrors which conserve flux in the annulus between Lyot stops. This result allows us to achieve the contrast necessary to observe of Earthlike exoplanets, while preserving most of the planet's flux.

\section{IMPLEMENTATION USING PUPIL REMAPPING OPTICS}

\subsection{Calculations}

We now explore replacing a transmissive apodizing mask with shaped mirrors, as depicted schematically in Figure 2. In order for this result to be practical and manufacturable, we want to minimize the complexity and 
the curvature of these mirrors. If we wish to use DMs, then the amplitude of the mirror deformation is limited as well. Based on our characterization of the mirror shapes for our apodization function without a spot in the vortex mask in section 4 , we expect the apodization with a spot will be conducive to being produced by mirror shapes that are relatively simple to manufacture.

Mirror shapes $h(r)$ and $\tilde{h}(\tilde{r})$ that produce the apodization function $A(r)$ are found by solving the MongeAmpere equation. ${ }^{2,33}$ While the mirror shapes found by solving Equations 1-3 above are useful for making qualitative statements about the mirrors, the treatment we provide below is equivalent to performing the ray tracing on each mirror separately. This ensures that we know each mirror separately as a function of the apodization function.

For a system of two shaped or deformable mirrors, the shape of the first mirror in the optical train, $h(r)$ to $A(r)$ is the forward Monge-Ampere equation,

$$
A(x, y)^{2}=\left(1+Z \frac{\partial^{2} h}{\partial x^{2}}\right)\left(1+Z \frac{\partial^{2} h}{\partial y^{2}}\right)-\left(Z \frac{\partial^{2} h}{\partial x \partial y}\right)^{2},
$$

where $Z$ is the distance between the first and second mirror. The second mirror shape is determined by the inverse equation,

$$
\frac{N}{A(\tilde{x}, \tilde{y})^{2}}=\left(1+Z \frac{\partial^{2} h}{\partial \tilde{x}^{2}}\right)\left(1+Z \frac{\partial^{2} h}{\partial \tilde{y}^{2}}\right)-\left(Z \frac{\partial^{2} h}{\partial \tilde{x} \partial \tilde{y}}\right)^{2} .
$$

The constant $N=\left(\int_{\text {Surface }} A(x, y)^{2} d S\right)^{2}$, which conserves the energy propagated between the two mirrors. The inverse equation can found by using the relationship between the remapping of light rays between the two mirrors (equation 22 in Pueyo \& Norman ${ }^{2}$ ) to convert between $h$ and $\tilde{h}$. In radial coordinates, these equations are expressed as

$$
\begin{aligned}
& A(r)^{2}=\frac{1}{r}\left(r+Z \frac{\partial h}{\partial r}\right)\left(1+Z \frac{\partial^{2} h}{\partial r^{2}}\right) \\
& \frac{N}{A(\tilde{r})^{2}}=\frac{1}{\tilde{r}}\left(\tilde{r}+Z \frac{\partial \tilde{h}}{\partial \tilde{r}}\right)\left(1+Z \frac{\partial^{2} \tilde{h}}{\partial \tilde{r}^{2}}\right)
\end{aligned}
$$

Because of the choice of normalization in the second Monge-Ampere equation, these mirror shapes conserve energy inside the entire telescope aperture, assuming that there is no central obscuration. However, in practice, this means that there will be some throughput loss as light is scattered from the pupil into the region blocked by the central Lyot stop. For an 0.3R obscuration and 0.45R inner Lyot stop, the throughput loss is about 0.04. Given that conserving the energy incident in the annulus between the Lyot stops requires a mirror shape of greater curvature, and that this is only a marginal loss in throughput, we elect to allow for this loss in throughput. Our mirror design allows for an off-axis throughput of $\sim 0.75$ after accounting for the Lyot stops with a real IWA that is still $\sim 1.5 \lambda / D$. This design is a promising candidate for earthlike exoplanet characteristics, since not only does it produce an adequately deep dark hole but it preserves the detectable exoplanet flux remarkably well.

\subsection{Fitting to Mirror Shapes}

After solving the Monge-Ampere equations numerically for a pair of mirror shapes, we use these shapes to recalculate the PSF. We ensure that they reproduce the desired PSF. Next, we fit the mirror shapes with a finite set of basis functions to determine how many degrees of freedom we need to describe these shapes accurately enough to reproduce the PSF. We hope to be able to fit the mirror shape with as few modes and as low order modes in our basis set as possible, since this will result in the easiest prescription to actually manufacture.

Since the Monge-Ampere equation is a second order non-linear differential equation, we opt to find the best fit second derivatives of the mirror shapes with respect to radius, and we choose basis functions for the mirror 

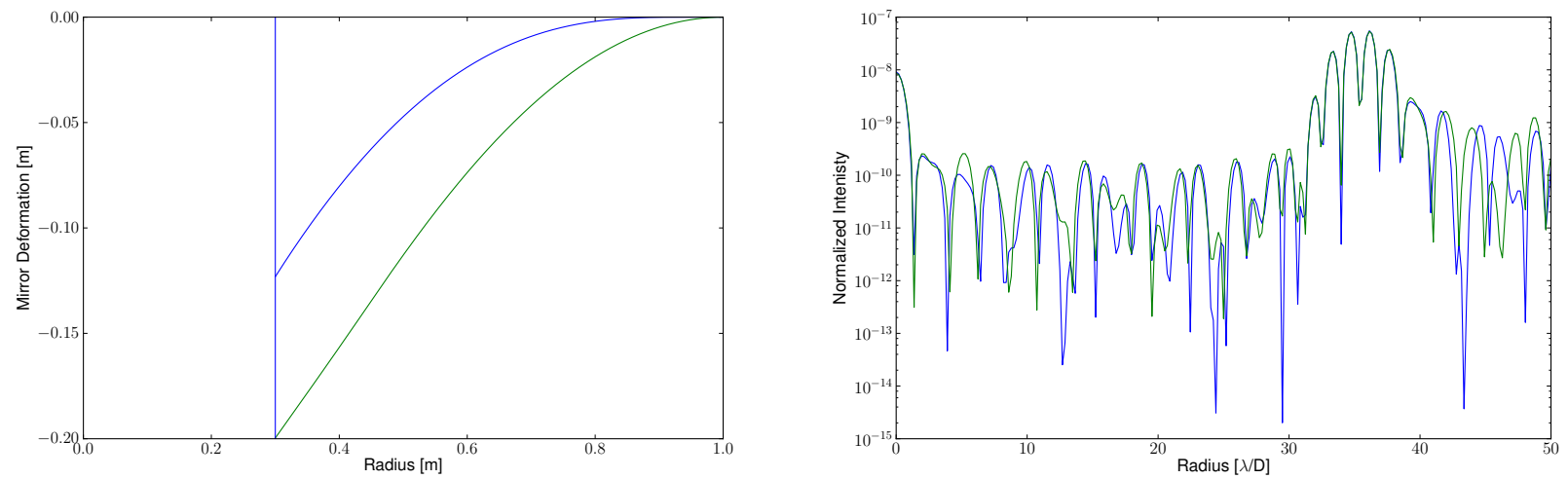

Figure 7. Left: Shapes of the two mirrors required to reproduce the apodization function in Figure 5. These mirror shapes were solved independently using the Monge-Ampere equations (Equations 15 and 16) in the region that is not covered by the central obscuration. The first mirror (upper curve) requires 35 Bessel DOFs to specify, and the second mirror (lower curve) requires 40. We do not contrain the centrally obscured region. The mirror shapes produced using the Monge-Ampere equations are consistent with the single-directional ray-mapping of the mirror shapes shown in Figure 4, although flipped. Right: PSF calculated by recalculating the apodization from the Monge-Ampere equations for each of the two mirrors independently. The apodization and resultant PSF for each mirror are essentially identical, and recreate the dark hole in Figure 5, so we are confident that the detail we have used to specify these mirror shapes is adequate for our coronagraph design.

shapes that have well behaved first and second derivatives. These basis functions are fourth order radial bessels, defined by

$$
F_{n}(r)=\frac{\sqrt{2} J_{4}\left(\frac{Z_{n} r}{R}\right)}{R J_{5}\left(Z_{n}\right)}
$$

where $Z_{n}$ is the nth fourth order Bessel zero. For the purposes of describing mirror shapes for manufacture, it may be more convenient to write the mirror shapes as an expansion of Zernike polynomials, but currently we are only exploring how complicated the mirror shapes need to be.

Bessel-expanded mirror shapes and the resulting PSF are shown in Figure 7. To reproduce the PSF from section 4.1, we needed 35 Bessel modes for the first mirror (blue in the figure above), and 40 Bessel modes for the second mirror (green). The PSF from the expanded mirror shapes vary little from the 'ideal' PSF shown in Figure 5- at the most divergent point in the dark hole the Bessel expanded mirrors fare worse than the ideal by only marginally. This result is encouraging, since we expect mirror shapes requiring $\sim 40$ degrees of freedom to be tractable to either manufacture or reproduce with DMs. In fact, the limiting factor on the strategy we pursue to construct these mirrors is the diffraction properties of the mirrors. Diffraction propagation depends on both the radius of the circularly symmetric mirror shapes and the distance between them. Since the amplitude of the mirror shapes go as $R^{2} / Z,{ }^{3,33}$ we will only be able to produce the dark hole with DMs if we can adequately control diffraction in small enough mirrors. Assuming a distance of $Z=1 \mathrm{~m}$, this implies that we will need to control diffraction for mirrors with $\sim 1 \mathrm{~cm}$ radius if we are to use DMs.

\subsection{Diffraction}

We studied the effects of diffraction on these mirror shapes using the S-Huygens approximation of Vanderbei et al. $2006 .{ }^{34}$ In particular, we used Equation 24 of this paper to calculate the diffractive propagation of light across the two mirror shapes. S-Huygens is a similar approximation to the Fresnel approximation of the integral describing Huygens wavelet propagation; the key difference being that the large separation and thin lens approximations are abandoned in favor of simplifying the azimuthal dependence of the integral in a way that preserves ray-optics. While the Fresnel approximation is accurate in a regime where the reflecting surface has small (approximately linear) perturbations from flatness, S-Huygens more accurately approximates the propagation of light reflected from highly deformed surfaces. 

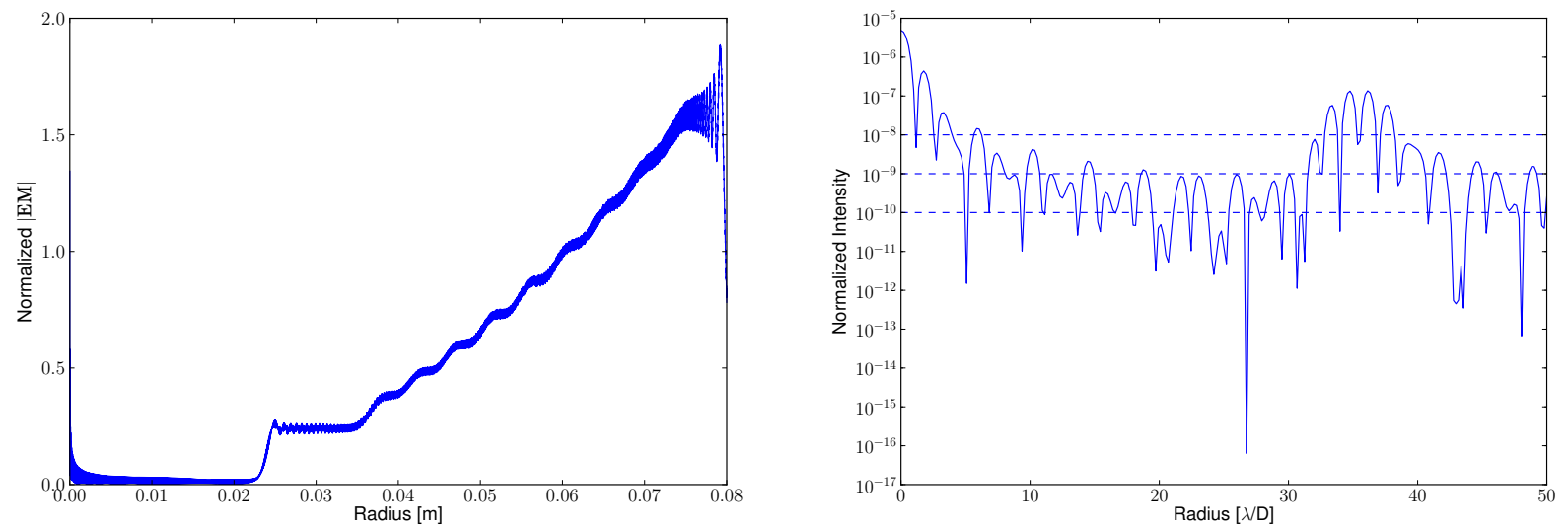

Figure 8. Left: Amplitude of the electric field at the pupil plane taking into account the effects of diffraction. We assume that our setup consists of two $8 \mathrm{~cm}$ mirrors $1 \mathrm{~m}$ apart. This figure is analogous to the throughput curves in Figures 3 and 5 , and is the normalized electric field amplitude in the sense that the on-axis point source flux is taken to be 1. Right: Loss of contrast ratio in the dark hole due to diffraction is shown in this panel. The three horizontal dashed lines correspond to normalized intensities of $10^{-8}, 10^{-9}$, and $10^{-10}$. Except near the designed-for IWA, the contrast ratio in the dark hole does better than $10^{8}$, and we expect to recover an $\sim 10^{10}$ contrast ratio using strategies to mitigate diffraction.

We chose this approximation to describe diffraction in our mirrors because, while the outer portion of the mirror shape becomes approximately flat, near the secondary obscuration the mirror shapes for both the first and second mirror deviates significantly from a linear approximation. However, it is worth emphasizing that a Fresnel treatment of the propagation is essentially valid near the outer edge of the mirror. This will open up a number of possibilities for compensating for diffraction.

The best results we had for degradation of the PSF due to diffraction were obtained assuming $8 \mathrm{~cm}$ optics $1 \mathrm{~m}$ apart. The diffracted electric field at the pupil plane and the final PSF are shown in Figure 8. As is apparent, diffraction in this system substantially degrades the contrast ratio in the dark hole. However, the resulting average contrast in the dark hole of $>10^{8}$ is still useful for planet imaging, and we have not yet made any attempt to compensate for diffraction. Compensation can be achieved through post-apodizers (e.g. PIAACMC,${ }^{5}$ or, owing to the flatness of the mirror shapes near the pupil edge, by oversizing the optics we use. ${ }^{35}$ In an upcoming paper we will explore mitigation strategies for diffraction on these mirror shapes. Furthermore, while the deformation necessary to produce our mirror shapes in optics this size are not feasible for DMs, we will also attempt to apply these strategies on smaller optics, and address the issue of using DMs to produce our coronagraph then.

\section{DISCUSSION}

The apodizer mask design shown in figure 3 is noteworthy due to its simplicity, and the simplicity of mirror shapes needed to produce it when implemented with PIAA-like mirrors. The necessary mirror shapes, determined using the calculations summarized in section 2.3, are shown in figure 5. While our coronagraph design may be achieved with a classical apodizer, we surmise that these results pave the way for a feasible design using shaped or deformable mirrors which will have superior throughput characteristics for off axis sources (e.g Earthlike exoplanets).

When we explored this design in more detail, we found that we were able to produce a variant on our pupil apodized vortex coronagraph with potentially improved robustness to tip/tilt and extended angular size of the on-axis source. We found that the apodization required to achieve a dark hole suitable for viewing an exo-Earth could be achieved with mirror shapes that can be described with $\leq 40$ basis functions, as well. This implies a coronagraph design using mirrors is highly manufacturable, and a design using deformable mirrors is plausible. 


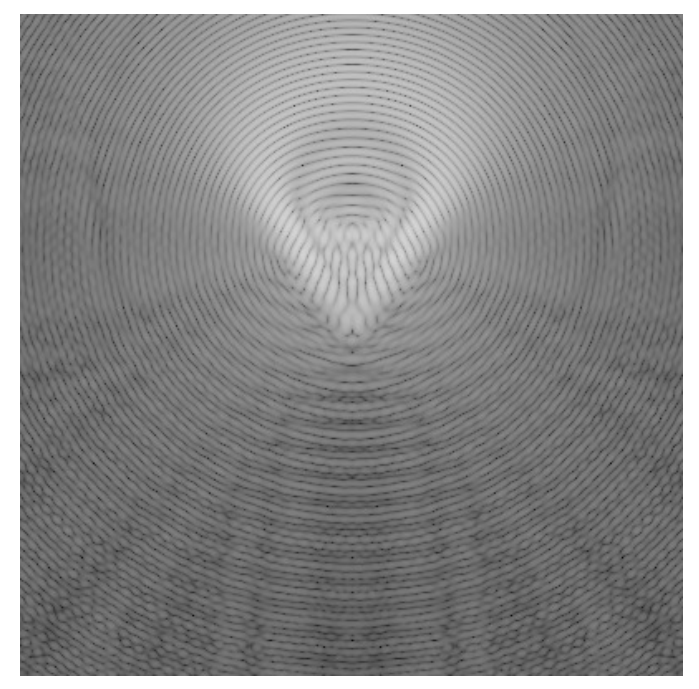

Figure 9. An example of the PSF of an off-axis source propagated through our coronagraph design using shaped mirrors. The smear on the PSF removes any clearly defined spatial peak in the image, so it will be necessary to employ reserve aspheric optics to reconstruct the undistorted image of the exoplanet.

The main issues moving forward with mirrors are the $R^{2} / Z$ dependence of the mirror deformation amplitude and the effects of diffraction. Without trying to mitigate the effects of diffraction, our best dark hole is achieved using $8 \mathrm{~cm}$ mirrors. The required deformation would be on the order of a mm, which far exceeds the stroke of current AO systems. Even this uncorrected design has potentially useful applications with shaped mirrors, since the contrast ratio it achieves does better than $10^{8}$. In a subsequent paper, we will address these problems by applying oversized optics to dampen diffraction at the mirrors' outer edges, or by using post-apodizers. The last remaining concern with our mirror design is smearing of the off-axis PSF (see Figure 9). Reconstruction of the PSF will require reverse aspheric optics to be placed downstream of the coronagraph; this technology has been well studied in PIAA coronagraph designs. ${ }^{5}$

The coronagraph we discuss in this paper can be used in conjunction with ACAD. ACAD is designed to be able to address the challenges of high-contrast imaging with large on-axis telescopes, which include ELTs in general. Spiders are compensated for on ACAD using a system of two deformable mirrors. The deformations of these mirrors match the spiders in the telescope aperture using solutions to the Monge-Ampere equation, and greatly reduce their effect on telescope PSF within a specified dark hole. ${ }^{2}$ With the spiders compensated for, a coronagraph design which accounts for a circularly symmetric pupil affected by a central obscuration can deliver extreme high contrast in the dark hole.

In the original ACAD paper Pueyo and Norman used a coronagraph with apodization and an opaque mask. This coronagraph was not optimal in terms of throughput, IWA, and contrast. However, ACAD can work in principle with any coronagraph, and we have developed an optimal design that compensates for the secondary mirror.

Since both mirrors vary smoothly and with low curvature, it is conceivable that the mirror shapes can be achieved using the deformable mirrors in the ACAD AO system if we can address the problem of diffraction on small enough optics. In its current state, our design is ready to be implemented using a classical apodizer, and shows considerable promise if suitable shaped mirrors are manufactured. We have shown a coronagraphic design capable of meeting the requirements to image Earthlike planets that can be incorporated into existing AO systems comprised of existing technologies, and can be used in conjunction with a system compensating for spiders with ACAD. With further study, we intend to examine the incorporation of this coronagraphic design into the ACAD concept, and consider how this system could be applied to upcoming telescopes. 


\section{ACKNOWLEDGMENTS}

This work was carried out in part for the Fourier Optics course (AS.171.755), and I am grateful for the guidance of course instructors Ron Allen and Anand Sivaramakrishnan.

This material is also partially based upon work carried out under subcontract 1496556 with the Jet Propulsion Laboratory funded by NASA and administered by the California Institute of Technology.

\section{REFERENCES}

[1] Martinez, P., Vernet, E., Dorrer, C., Aller Carpentier, E., Boccaletti, A., Kasper, M., Baudrand, J., and Chaumont, C., "Phase and Lyot-type coronagraphs for the High Order Testbench: prototyping and first laboratory results," in [Society of Photo-Optical Instrumentation Engineers (SPIE) Conference Series], Society of Photo-Optical Instrumentation Engineers (SPIE) Conference Series 7015 (July 2008).

[2] Pueyo, L. and Norman, C., "High Contrast Imaging with an Arbitrary Aperture: Active Correction of Aperture Discontinuities," ArXiv e-prints (Nov. 2012).

[3] Guyon, O., "Phase-induced amplitude apodization of telescope pupils for extrasolar terrestrial planet imaging," A\&A 404, 379-387 (June 2003).

[4] Soummer, R., Sivaramakrishnan, A., Pueyo, L., Macintosh, B., and Oppenheimer, B. R., "Apodized Pupil Lyot Coronagraphs for Arbitrary Apertures. III. Quasi-achromatic Solutions," ApJ 729, 144 (Mar. 2011).

[5] Guyon, O., Hinz, P. M., Cady, E., Belikov, R., and Martinache, F., "High Performance Lyot and PIAA Coronagraphy for Arbitrarily Shaped Telescope Apertures," ApJ 780, 171 (Jan. 2014).

[6] Quirrenbach, A., "Coronographic Methods for the Detection of Terrestrial Planets," ArXiv Astrophysics e-prints (Feb. 2005).

[7] Guyon, O., "Limits of Adaptive Optics for High-Contrast Imaging," ApJ 629, 592-614 (Aug. 2005).

[8] Mawet, D., Pueyo, L., Lawson, P., Mugnier, L., Traub, W., Boccaletti, A., Trauger, J. T., Gladysz, S., Serabyn, E., Milli, J., Belikov, R., Kasper, M., Baudoz, P., Macintosh, B., Marois, C., Oppenheimer, B., Barrett, H., Beuzit, J.-L., Devaney, N., Girard, J., Guyon, O., Krist, J., Mennesson, B., Mouillet, D., Murakami, N., Poyneer, L., Savransky, D., Vérinaud, C., and Wallace, J. K., "Review of small-angle coronagraphic techniques in the wake of ground-based second-generation adaptive optics systems," in [Society of Photo-Optical Instrumentation Engineers (SPIE) Conference Series], Society of Photo-Optical Instrumentation Engineers (SPIE) Conference Series 8442 (Sept. 2012).

[9] Trauger, J., Stapelfeldt, K., Traub, W. A., Krist, J., Moody, D., Serabyn, E., Mawet, D., Pueyo, L., Shaklan, S., Henry, C., Park, P., Gappinger, R., Brugarolas, P., Alexander, J., Mireles, V., Dawson, O., Guyon, O., Kasdin, J., Vanderbei, B., Spergel, D., Belikov, R., Marcy, G., Brown, R., Schneider, J., Woodgate, B., Matthews, G., Egerman, R., Voyer, P., Vallone, P., Elias, J., Conturie, Y., Polidan, R., Lillie, C., Spittler, C., Lee, D., Hejal, R., Bronowick, A., Saldivar, N., Ealey, M., and Price, T., "ACCESS: A Concept Study for the Direct Imaging and Spectroscopy of Exoplanetary Systems," in [Pathways Towards Habitable Planets], Coudé Du Foresto, V., Gelino, D. M., and Ribas, I., eds., Astronomical Society of the Pacific Conference Series 430, 375 (Oct. 2010).

[10] Trauger, J. T. and Traub, W. A., "A laboratory demonstration of the capability to image an Earth-like extrasolar planet," Nature 446, 771-773 (Apr. 2007).

[11] Trauger, J., Moody, D., Gordon, B., Krist, J., and Mawet, D., "A hybrid Lyot coronagraph for the direct imaging and spectroscopy of exoplanet systems: recent results and prospects," in [Society of Photo-Optical Instrumentation Engineers (SPIE) Conference Series], Society of Photo-Optical Instrumentation Engineers (SPIE) Conference Series $\mathbf{8 1 5 1}$ (Sept. 2011).

[12] Foo, G., Palacios, D. M., and Swartzlander, Jr., G. A., "Optical vortex coronagraph," Optics Letters 30, 3308-3310 (Dec. 2005).

[13] Mawet, D., Serabyn, E., Wallace, J. K., and Pueyo, L., "Improved high-contrast imaging with on-axis telescopes using a multistage vortex coronagraph," Optics Letters 36, 1506 (Apr. 2011).

[14] Mawet, D., Pueyo, L., Carlotti, A., Mennesson, B., Serabyn, E., and Wallace, J. K., "Ring-apodized Vortex Coronagraphs for Obscured Telescopes. I. Transmissive Ring Apodizers," ApJS 209, 7 (Nov. 2013). 
[15] Vanderbei, R. J. and Traub, W. A., "Pupil Mapping in Two Dimensions for High-Contrast Imaging," ApJ 626, 1079-1090 (June 2005).

[16] Cady, E., "Design of Mirrors and Apodization Functions in Phase-induced Amplitude Apodization Systems," ApJs 201, 25 (Aug. 2012).

[17] Martinez, P., Dorrer, C., Aller Carpentier, E., Kasper, M., Boccaletti, A., Dohlen, K., and Yaitskova, N., "Design, analysis, and testing of a microdot apodizer for the Apodized Pupil Lyot Coronagraph," A\&SA 495, 363-370 (Feb. 2009).

[18] Mawet, D., Riaud, P., Absil, O., and Surdej, J., "Annular Groove Phase Mask Coronagraph," ApJ 633, 1191-1200 (Nov. 2005).

[19] Mawet, D., Riaud, P., Hanot, C., Vandormael, D., Loicq, J., Baudrand, J., Surdej, J., and Habraken, S., "The annular groove phase mask coronagraph: an achromatic optical vortex," in [Society of Photo-Optical Instrumentation Engineers (SPIE) Conference Series], Society of Photo-Optical Instrumentation Engineers (SPIE) Conference Series 6693 (Sept. 2007).

[20] Mawet, D., Trauger, J. T., Serabyn, E., Moody, Jr., D. C., Liewer, K. M., Krist, J. E., Shemo, D. M., and O'Brien, N. A., "Vector vortex coronagraph: first results in the visible," in [Society of Photo-Optical Instrumentation Engineers (SPIE) Conference Series], Society of Photo-Optical Instrumentation Engineers (SPIE) Conference Series $\mathbf{7 4 4 0}$ (Aug. 2009).

[21] Mawet, D., Serabyn, E., Moody, D., Kern, B., Niessner, A., Kuhnert, A., Shemo, D., Chipman, R., McClain, S., and Trauger, J., "Recent results of the second generation of vector vortex coronagraphs on the high-contrast imaging testbed at JPL," in [Society of Photo-Optical Instrumentation Engineers (SPIE) Conference Series], Society of Photo-Optical Instrumentation Engineers (SPIE) Conference Series $\mathbf{8 1 5 1}$ (Sept. 2011).

[22] Murakami, N., Nishikawa, J., Traub, W. A., Mawet, D., Moody, D. C., Kern, B. D., Trauger, J. T., Serabyn, E., Hamaguchi, S., Oshiyama, F., Sakamoto, M., Ise, A., Oka, K., Baba, N., Murakami, H., and Tamura, M., "Coronagraph focal-plane phase masks based on photonic crystal technology: recent progress and observational strategy," in [Society of Photo-Optical Instrumentation Engineers (SPIE) Conference Series], Society of Photo-Optical Instrumentation Engineers (SPIE) Conference Series 8442 (Sept. 2012).

[23] Serabyn, E., Trauger, J., Moody, D., Mawet, D., Liewer, K., Krist, J., and Kern, B., "High-contrast imaging results with the vortex coronagraph," in [SPIE Optical Engineering+ Applications], 88640Y-88640Y, International Society for Optics and Photonics (2013).

[24] Mawet, D., Pueyo, L., Moody, D., Krist, J., and Serabyn, E., "The Vector Vortex Coronagraph: sensitivity to central obscuration, low-order aberrations, chromaticism, and polarization," in [Society of Photo-Optical Instrumentation Engineers (SPIE) Conference Series], Society of Photo-Optical Instrumentation Engineers (SPIE) Conference Series $\mathbf{7 7 3 9}$ (July 2010).

[25] Jenkins, C., "Optical vortex coronagraphs on ground-based telescopes," MNRAS 384, 515-524 (Feb. 2008).

[26] Soummer, R., "Apodized Pupil Lyot Coronagraphs for Arbitrary Telescope Apertures," ApJL 618, L161L164 (Jan. 2005).

[27] Traub, W. A. and Vanderbei, R. J., "Two-Mirror Apodization for High-Contrast Imaging," ApJ 599, 695701 (Dec. 2003).

[28] Martinache, F., Guyon, O., Clergeon, C., Garrel, V., and Blain, C., "The Subaru coronagraphic extreme AO project: first observations," in [Society of Photo-Optical Instrumentation Engineers (SPIE) Conference Series], Society of Photo-Optical Instrumentation Engineers (SPIE) Conference Series $\mathbf{8 4 4 7}$ (July 2012).

[29] Pluzhnik, E. A., Guyon, O., Ridgway, S. T., Martinache, F., Woodruff, R. A., Blain, C., and Galicher, R., "Exoplanet Imaging with a Phase-induced Amplitude Apodization Coronagraph. III. Diffraction Effects and Coronagraph Design," ApJ 644, 1246-1257 (June 2006).

[30] Lyot, B., "The study of the solar corona and prominences without eclipses (George Darwin Lecture, 1939)," MNRAS 99, 580 (June 1939).

[31] Sivaramakrishnan, A., Koresko, C. D., Makidon, R. B., Berkefeld, T., and Kuchner, M. J., "Ground-based Coronagraphy with High-order Adaptive Optics," ApJ 552, 397-408 (May 2001). 
[32] Carlotti, A. and Pueyo, L., "Influence of surface errors on the design of PIAA mirrors using numerical and semi-analytical propagation models," in [Society of Photo-Optical Instrumentation Engineers (SPIE) Conference Series], Society of Photo-Optical Instrumentation Engineers (SPIE) Conference Series $\mathbf{8 1 5 1}$ (Sept. 2011).

[33] Pueyo, L., Kasdin, N. J., Carlotti, A., and Vanderbei, R., "Design of Phase Induced Amplitude Apodization Coronagraphs Over Square Apertures," ApJS 195, 25 (Aug. 2011).

[34] Vanderbei, R. J., "Diffraction Analysis of Two-dimensional Pupil Mapping for High-Contrast Imaging," ApJ 636, 528-543 (Jan. 2006).

[35] Pueyo, L. and Kasdin, N. J., "Polychromatic Compensation of Propagated Aberrations for High-Contrast Imaging," ApJ 666, 609-625 (Sept. 2007). 